\title{
Les créoles français sont-ils des langues néo-romanes?
}

\author{
Marie E. Paul \\ Université Paris III, Tulane University
}

L'unique consensus pour définir les langues créoles est la prise en compte des éléments sociohistoriques que R. Chaudenson (2003) désigne comme constituant l'état civil : le lieu (majoritairement des îles), la date (Le XVII ${ }^{\mathrm{e}}$ et le $\mathrm{XVIII}^{\mathrm{e}}$ siècles) et les parents (les langues en contact). Les traits typologiques proposés ne semblent guère satisfaire les créolistes et il ne parait pas plus facile de les classer généalogiquement. Nous nous sommes penchée sur cette question parce qu'il ressort que la question de la filiation des langues créoles pose encore problème alors que le débat a été lancé, il y a plus d'un siècle. Notre intervention n'a pas la prétention de couvrir de manière détaillée l'ensemble des créoles français mais de proposer un ensemble de réflexions qui traiteront du cas précis du créole haïtien tout en faisant référence parfois aux créoles antillais. Nous pensons qu'à partir de ces cas précis la question peut être étendue à l'ensemble des créoles français voire romans.

Ainsi passerons-nous en revue dans un premier temps les différentes voix qui se sont exprimées sur la filiation des créoles français, notamment celui d'Haiti. Nous verrons ensuite la méthode préconisée et utilisée par la linguistique historique pour les langues naturelles en général ${ }^{1}$. Enfin, il s'agira d'observer si elle a été appliquée jusque là. Si oui pourquoi cette cacophonie persiste-elle ? Si non pourquoi ?

\section{De la difficulté d'établir la filiation généalogique des langues créoles}

Les créoles français se divisent en deux groupes : ceux de la zone américano-caraïbe et ceux de l'Océan Indien. Ils sont parlés par un peu plus de 10 millions de personnes et leur étude a commencé dès la seconde moitié du XIX $\mathrm{X}^{\mathrm{e}}$ siècle avec les travaux de H. Schuchardt (1880), L. Adam (1883). Celui-ci dans son ouvrage intitulé Essai d'hybridologie linguistique, désigne respectivement les créoles guyanais et mauriciens comme des idiomes négro-aryen et maléo-aryen. Depuis, la question de la filiation de ces langues ne cesse d'être posée : elle surgit en Haïti de manière discordante en 1936 par le biais de Suzanne Comhaire-Sylvain et de Jules Faine. Suzanne Sylvain (1936) établi des ressemblances entre les langues africaines et le créole haïtien. Dans la même année, comme pour prendre le contre-pied des travaux de Sylvain, Jules Faine défend la thèse opposée, celle de l'étymologie française, voire normande du créole haïtien. Onze ans après, Charles Fernand Pressoir (1947: 10) conforte la thèse dite substratiste dans Débats sur le créole et le folklore en réaffirmant que le «créole est une langue mixte ayant à sa base le vocabulaire français agencé selon les principes des grammaires africaines...»

En France, des linguistes tels qu'A. Meillet (1921) et F. Brunot (1936) affirmeront la filiation française. Brunot n'hésite pas à classer les créoles français dans la droite lignée de la langue française dans son Histoire de la langue française. Quelque 14 ans plus tôt, A. Meillet (1921) s'exprimait déjà en ces termes : «Le créole de la Réunion ou de la Martinique est du français imparfait [...] Il subsiste toujours dans le système nouveau une portion notable du système ancien et le peu que le créole a de grammaire est de la grammaire française. » Au Canada, Claire Lefebvre (1998) reprendra la position dite substratiste.

En 2006, S. Mufwene relance le débat en posant la question du classement généalogique des langues créoles dans son article «Les créoles : de nouvelles variétés indo-européennes désavouées ? » paru dans les actes du colloque Créolisation linguistique et Sciences humaines aux Presses Universitaires HaïtianoAntillaises.

Ce panorama dit à quel point il est difficile de définir et de classer les langues créoles. Elles semblent diverger de la lignée des langues parentes et apparaissent comme une catégorie à part (I. Dyen, J. Kruskal \& P. Black1992). 


\section{La méthode de la grammaire comparée}

Parcourons les œuvres majeures de deux linguistes de référence en linguistique historique : A. Meillet et A. Schleicher et relevons les étapes de la méthode comparée. Dans le chapitre traitant de la définition de la méthode, Meillet explique que la linguistique historique se base (1) sur la philologie car elle met à sa disposition les textes anciens attestant de suites d'états de la langue mais elle ne peut s'y limiter. Le linguiste doit également établir des correspondances : «pour tous les groupes actuellement établis et étudiés d'une manière méthodique, le moyen de faire le rapprochement est de (2) poser une «langue commune » initiale. Pour poser cette langue commune (3), il faut retrouver dans les langues comparées les traits spécifiques de cette langue. En ce qui a trait à la morphologie, l'auteur affirme qu'il n'est pas recommandé de faire usage de ces types généraux (telle la flexion) pour démontrer une «parenté de langues ", car il arrive souvent que, avec le temps, le type tende à s'effacer plus ou moins complètement, comme l'histoire des langues indo-européennes le montre. Pour Meillet, ce qui est probant pour établir la continuité entre une langue commune et une langue ultérieure, ce sont les procédés particuliers d'expression de la morphologie. Il cite en exemple l'usage de de ou 's pour exprimer le rapport de dépendance entre deux substantifs mais souligne aussitôt que l'emploi de de peut disparaître d'un parler français ou celui de 's d'un parler anglais sans que pour cela, ces parlers cessent d'être français ou anglais et il conclut que seuls les faits positifs ont une valeur probante. Le linguiste fait observer que les différences relevées d'un système phonétique à un autre ne sont pas capricieuses, elles obéissent à une régularité. Toutefois, il est fort difficile sinon impossible de mettre en évidence les correspondances régulières entre deux langues provenant de la langue mère. En revanche les correspondances entre la langue commune et chacune de ses langues filles obéissent à des régularités, c'est ce qu'on appelle « lois phonétiques $\gg$.

Pour ce qui a trait au lexique, étant donné que c'est l'élément le plus sujet au changement, les rapprochements étymologiques devraient se faire d'après les règles de correspondances et non d'après la forme phonétique. Qu'il s'agisse de morphologie, de phonétique ou de vocabulaire, en dépit de tout le principe à suivre est la soumission des rapprochements à des règles strictes. A. Schleicher (1852: 37) rappelle à plusieurs reprises dans sa partie consacrée à la méthodologie que l'établissement de la parenté généalogique ne peut être basée sur de simples ressemblances mais qu'il faut établir les lois phonétiques :

\footnotetext{
Avant de proclamer un mot comme identique dans deux langues, il nous faudra l'analyser grammaticalement, le disséquer comme font les anatomistes, le décomposer comme font les chimistes. Il nous faudra savoir comment, d'après les lois phonétiques de ces deux idiomes, le mot en question doit être formé dans chacun.
}

Lorsque nous comparons l'état de la recherche sur les langues créoles et la méthodologie préconisée par Schleicher et Meillet dans la méthode comparative, il nous semble que l'ensemble des précautions ont été observées : l'usage des documents anciens (M-C Hazaël-Massieux 2008, Guy Hazaël- Massieux 1996), la langue commune (la koinè des colons, dont les parlers français de l'Amérique du nord ont conservé beaucoup de caractéristiques (cf. H. Wittman 1994, R. Mougeon \& E. Beniak 1994), l'établissement de correspondances au sein du vocabulaire (Bernabé 2002), de la phonétique (Thèse de Fabrice Delumeau 2006), quant à la morphologie, la thèse de Delumeau révèle des correspondances au plan de la morphologie du nom. En ce qui concerne la morphologie du verbe, un créole tel que celui de Haïti ou ceux des petites Antilles n'ont pas gardé la morphologie flexionnelle du verbe. A ce sujet, Meillet et Schleicher affirment que seuls les éléments positifs (ceux qui sont conservés) importent.

De plus, nous pourrions ajouter - pour corroborer notre ensemble de réflexions - que beaucoup ont reconnu les similitudes dans le mode de développement du latin au français et du français aux créoles : Schlieben-Lange, B. (1977) Goyette, S. (2001), Fon Sing, G. (2006). Le contact est un élément fondamental dans le développement de ces langues. Cependant les langues créoles ont souvent été qualifiées de langues mixtes sans que cette désignation soit clairement expliquée. 


\title{
3 Qu'est ce qu'une langue mixte, si cela existe ?
}

Dans la réédition de 1952 des Langues du monde d'Antoine Meillet et Marcel Cohen, les langues créoles sont traitées à deux niveaux. Premièrement, dans la section des langues indo-européennes, à la suite des parlers romans, elles sont traitées par J. Vendryes et E. Benveniste comme des langues issues du contact de langues romanes et de langues indigènes ou importées :

\begin{abstract}
Sur plusieurs points du globe, le portugais, l'espagnol et le français, en contact avec des langues indigènes ou importées (Antilles), ont produit divers parlers créoles, sortes de langues mixtes dont plusieurs ont été assez bien étudiées : négro-portugais du Cap Vert et de la Guinée portugaise ; indo-portugais parlé à Ceylan par les descendants des colons, papiamento des nègres de Curaçao, créole de Haïti, de la Réunion, de l'ile Maurice, etc. (T. 1, p. 52)
\end{abstract}

Dans le second tome M. Delafosse et A. Caquot y font référence - dans la partie réservée aux langues de l'Afrique noire en appendice aux langues du Soudan-Guinée - en les traitant toujours comme des langues de contact et désignées par la double origine « le négro-français ou le négro-portugais » :

\begin{abstract}
A l'occasion de la traite des esclaves et à la suite des contacts qui en ont résulté entre les Européens et les Noirs, il s'est formé sur la côte occidentale d'Afrique des parlers mixtes qui ont acquis depuis une extension de plus en plus grande et qui sont employés couramment aujourd'hui par les Européens et les indigènes dans les relations mutuelles et même souvent par les indigènes entre eux lorsqu'ils ont des langues maternelles différentes. (T. 2, p. 842)
\end{abstract}

Trois choses sont à relever, ces langues sont désignées comme des langues de contact, des langues mixtes et la double parenté est désignée. La rédaction de ces articles date de 1929 (date de la première édition) à 1950 à peu près (la seconde édition ayant paru en 1952, à cause d'interruptions). Nous retrouvons le qualificatif «mixte» pour désigner les langues créoles alors que, chose étonnante, A. Schleicher, luimême, a nié l'existence de langues mixtes en 1852 dans son ouvrage Les langues de l'Europe moderne p. 38 : «il n'y a pas ce qu'on appelle une langue mixte, comme il n'y a pas d'organisme naturel mixte » avant d'ajouter: " L'influence des autres idiomes sur une langue ne se borne peut être pas à la sphère simplement lexicale ».

La position de Meillet (1925) dans le chapitre relatif aux langues mixtes est assez claire quoique nuancée: la notion de langue mixte, pour l'auteur, correspond au résultat observable sur 'la langue générale' après contact avec des langues indigènes, ce sont les influences du substrat sur la langue qui s'impose. Voici comment l'auteur résume son point de vue: «Une langue généralisée, est sur les domaines où elle s'étend, une langue plus ou moins mixte, puisqu'à coté d'une norme universelle, on y observe l'influence-sans doute profonde- d'usages locaux, d'habitudes locales » (p. 81). A la page 83, l'auteur dit qu'au moment où il rédige (sachant que l'ouvrage paraît en 1925) «il ne s'est pas rencontré jusqu'ici de cas où l'on ait été conduit à poser que le système morphologique d'une langue donnée résulte d'un mélange des morphologies de deux langues distinctes. Dans tous les cas observés jusqu'à présent, il y a une tradition continue d'une langue ».

Par prudence le linguiste reste ouvert et considère que si l'on venait à découvrir ce type de langue la tâche des linguistes pour malaisée qu'elle serait devrait très certainement les conduire à changer de méthode :

\footnotetext{
Néanmoins on ne saurait affirmer que dans certains cas favorables, il n'y ait pas eu des « mélanges » véritables. Le jour où l'on viendrait à les rencontrer, la tâche du linguiste serait malaisée. Si l'on a pu arriver à faire par la comparaison, l'histoire de quelques langues, c'est que l'on était sûr que chaque système nouveau devait s'expliquer en partant d'un système unique. Au cas où l'on devrait faire état de deux systèmes initiaux et de réactions de l'un sur l'autre les méthodes actuelles ne suffiraient pas. Car le droit qu'on aurait de choisir entre deux séries de formes originelles causerait un tel arbitraire que toute démonstration deviendrait presque irréalisable. Malgré les hypothèses faites en ce sens, les linguistes ne se sont par bonheur jamais jusqu'ici trouvés de manière sûre devant pareille difficulté. Pour en triompher, la linguistique
} 
devra, si la difficulté vient à se rencontrer vraiment, élaborer de nouvelles méthodes, plus délicates que celles qui sont décrites ici, et il resterait à les éprouver.

Les langues créoles constitueraient-elles cette variété inclassable par les méthodes actuelles et qui causeraient l'effondrement de l'édifice ? Cet ouvrage date de 1925 et à cette époque les langues créoles étaient connues, de ce fait il nous semble que Meillet ne considère pas ces langues comme mixtes.

F. Brunot en 1936, donc contemporain des auteurs de ces articles, s'exprimait sur le sujet en ces termes : tome VIII p. 1143 :

Ces parlers ne sont pas des langues mixtes, des mulâtres linguistiques; ce sont des dérivés, des provignements du français. Toutefois leur formation n'a pas eu lieu indépendamment des langues que les nègres avaient apportées d'Afrique. On ne peut pas tout expliquer par elles, on ne doit pas tout expliquer sans elles. Ces langues n'ont peut être pas fourni grand chose ni du matériel ni de la structure des formes ou des phrases. Si leur action positive ${ }^{2}$ 'a pas été très grande, leur action négative ${ }^{3}$ doit sans cesse entrer en considération.

Nous en arrivons à un point où nous devons nous interroger sur la raison de cette cacophonie sur « la mixité des langues créoles ». Schleicher, en tant que Hégélien et Darwinien mais linguiste, réfute l'existence de ce type de langues. F. Brunot fournit un élément d'explication à l'origine de cette désignation, la mixité tiendrait du fait que les populations locutrices des langues parentes soient de phénotype différent: "Ces parlers ne sont pas des langues mixtes, des mulâtres linguistiques ». Les auteurs, Vendryes et Benveniste, fourniraient-ils la confirmation de l'explication raciologique lorsque dans 'les généralités introductrices' ils font cette annotation qui aura retenu notre attention?

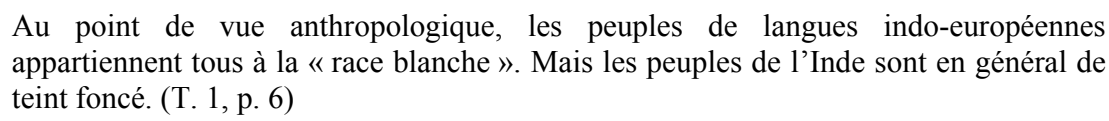

Pourquoi cette mise au point contradictoire? Quel est son sens, si ce n'est de justifier que les indoeuropéens sont de race blanche, ou sont à les considérer comme de race blanche alors qu'ils ne le sont pas tous de fait.

Pour en revenir à la notion de contact, en œuvre dans la définition des langues créoles mais également dans l'histoire des langues romanes, notons que Schleicher ne nie pas cette caractéristique aux langues indo-européennes :

N'oubliez pas non plus que toutes ces nations, les véritables pionniers et architectes de la civilisation humaine, se sont mises en contact permanent entre elles ; c'est encore là un motif, du moins accessoire, de la décroissance des idiomes primitifs. (1852:23-24)

Bien plus il va jusqu'à identifier d'autres éléments en cause dans le changement linguistique qu'il désigne malheureusement du terme péjoratif de « dégénérescence » :

Les grandes époques, ce qu'on pourrait appeler les cataclysmes des races et des sociétés, sont accompagnées d'une rapide décroissance des idiomes ; la migration des peuples vers l'empire romain était suivie d'une dégénérescence subite des langues romanes et germaniques. (1852:25)

R. Chaudenson a souligné ce fait dans le compte rendu des actes du colloque 'Créolisation linguistique et sciences humaines' traitant du caractère plutôt analytique ou synthétique de l'expression du futur dans la lignée des langues indo-européennes :

Je pense, au vu de ces données, à la fois linguistiques et socio-historiques, et vu le rôle de l'appropriation en situations exolingues dans la constitution de nouveaux idiomes, que les périodes de grandes mutations sociales (avec une hétérogénéité linguistique et des contacts de langues importants) donnent un rôle majeur aux formes analytiques, celles-ci étant plus facilement analysables et identifiables et sémantiquement plus perceptibles et plus pleines [...]. En revanche, les périodes de stabilité et de cohésion 
sociales plus grandes vont entraîner souvent la synthétisation de formes auparavant analytiques. (2006: 177)

Olivier Soutet répond à plusieurs des questions laissées en suspens par le comparatiste allemand. Alors que Schleicher insiste sur la profondeur de l'analyse qui doit être menée dans l'étude comparative il semble laisser entendre - en disant que «l'harmonie lexicale entre deux langues sans l'harmonie grammaticale, ne prouve rien »-que pour conclure à la parenté généalogique, il faut avoir démontré les analogies lexicales et grammaticales. Soutet rappelle la distinction entre l'évolution structurale et l'évolution phonétique : «L'évolution phonétique explique-t-il est aveugle, elle est de l'ordre de la loi. » L'évolution structurale, elle, est tendancielle « non strictement systématique, parfois aléatoire : elle ne relève pas d'une prédictibilité absolue.» Ainsi il opère une distinction entre l'histoire interne des continuités articulatoires et celle des discontinuités structurales car du point de vue des continuités articulatoires le français n'est que du latin continué alors que d'après les discontinuités structurales la rupture est radicale quoique la datation historique en soit compliquée. Soutet explique cette dichotomie entre d'une part les correspondances articulatoires qui se présentent sous forme de continuité et d'autre part les équivalences structurales qui se révèlent comme discontinuités par le fait que la structure de la langue fille est nécessairement nouvelle :

\begin{abstract}
Une langue construit très largement sa grammaire à partir de signes qu'elle hérite de sa langue mère au plan de la forme, mais auxquels elle attribue un autre rôle [...] l'histoire grammaticale d'une langue se laisse dans ces conditions décrire comme l'adaptation -progressive, lente, parfois hésitante et inachevée - à une structure nouvelle (celle de la langue fille) de signes originellement adéquats à une autre structure (celle de la langue mère). (2005: 53)
\end{abstract}

Quel enseignement pouvons-nous tirer des analyses de Soutet quant à la créolistique ? Cela expliquerait pourquoi un créole comme celui d'Haïti a effectivement une grammaire à base de signes du français mais qu'il s'agit d'une grammaire nouvelle. Les éléments qui vont entrer en ligne de compte lors de $l^{\prime}$ " attribution de ces rôles nouveaux » sont de deux ordres celui du « déterminisme du milieu » ${ }^{4}$ et celui $\mathrm{du}$ « déterminisme structural $»^{5}$. Pour expliquer le déterminisme du milieu, Soutet parle notamment des 'influences' articulatoires liées aux langues antérieurement parlées. S. Mufwene (2005) s'est prononcé sur le rôle des influences dans sa théorie du «Feature Pool ». Il définit le «Feature Pool » comme « l'arène où les variantes : formes et structures se retrouvent en compétition les unes avec les autres ».

Dans son ouvrage, The Ecology of language p. 4-6, Mufwene (2001) présente un schéma explicatif du feature pool en trois phases. Ce schéma est d'un double intérêt, car il montre à quel niveau les langues substratiques ont pu influencer l'apparition de certains traits dans le processus de restructuration qui va engendrer les créoles. Il révèle aussi en amont, que c'est par le même type de mécanisme que la koiné ${ }^{6}$ a émergé.

L'historien du français Ferdinand Brunot (1935: 1137) s'est exprimé également sur l'impact des influences des langues antérieures. Voici ce qu'il en dit :

$$
\begin{aligned}
& \text { Une question primordiale en effet se pose, celle de savoir si la déformation du français } \\
& \text { a été influencée et de quelle façon elle l'a été par les langages que les nègres esclaves } \\
& \text { ont apporté de leurs pays, ce que cette influence a pu y introduire d'une part, ce qu'elle } \\
& \text { a pu d'autre part en faire disparaître, en un mot quelle a été l'action positive et négative } \\
& \text { de ces langues. Sur l'influence positive, tout le monde est à peu près d'accord : les } \\
& \text { éléments d'origine entrés dans le créole français sont extrêmement peu nombreux, } \\
& \text { particulièrement dans le vocabulaire. Il y aurait à débattre sur la morphologie. Forme } \\
& \text { n'est pas matière. Le français peut fournir la seconde, alors que la première est d'esprit } \\
& \text { nègre. Un pluriel comme frè yé, dont nous parlerons plus loin, est fait de deux } \\
& \text { éléments français, frè (frère) et yé (eux). Mais l'idée de cet assemblage est totalement } \\
& \text { inconnue au français et se rencontre en langue africaine. La création ne peut pas être } \\
& \text { considérée comme française. }
\end{aligned}
$$

Nous voici au terme de nos réflexions et nous constatons que les études créoles manifestent l'ensemble des éléments préconisés par la méthode, que les langues créoles ont une évolution similaire au français en 
ce qui a trait au contact, pourtant elles ont été désignées comme « mixtes », désignation non attribuée à la langue mère. Plusieurs auteurs ont explicitement exposé l'argument raciologique qui soutiendrait cette désignation (Brunot, Vendryès \& Benveniste). Il a été reconnu que la notion de langue mixte n'existe pas. Par conséquent quel comportement adopter face à cette situation? Faut-il désormais désigner toutes les langues issues de contact linguistique comme créoles ou 'réintégrer', rattacher les créoles dans la droite lignée des langues européennes (par exemple) ? Aucune de ces solutions ne semble idéale. Dans le premier cas, l'acception du terme « créole » privilégierait l'aspect du contact, ce qui noierait la dimension sociohistorique propre de ces jeunes langues. La seconde consisterait à accepter de classer les créoles comme de nouvelles variétés indo-européennes, comme le propose, à juste titre, Mufwene. Cependant, pourquoi ne mentionner qu'un parent dans l'arbre généalogique de la langue ? Pourquoi ne parle-t-on que de langues romanes ou germaniques? R. Nicolaï (2000) a élaboré un ensemble de questionnement critique, que nous pensons pertinent, sur le classement des langues créoles et les limites du modèle de représentation arborescent tel que l'a présenté Schleicher, et s'est interrogé sur la possibilité de modifier cette dernière. G. Hazaël-Massieux (1996) propose de les classer parmi les langues afro-romanes tout en retenant le terme général de langues néo-romanes.

\section{Références bibliographiques}

Adam, L. (1883). Les idiomes négro-aryen et maléo-aryen : Essai d'hybridologie linguistique. Paris : Maisonneuve.

Arends, J. (1993). Towards a gradualist model of creolization. Francis Byrne and John Holm (eds) Atlantic meets Pacific, 317-380. Amsterdam and Philadelphia : Benjamins.

Arends, J., (1995). The early stages of creolization. Amsterdam : Benjamins.

Arends, J., (2002). La « dé-historisation » de la créologenèse. Etudes Créoles, ${ }^{\circ} 1$.

Baker, P., (1987). Combien il y a t-il eu de genèses créoles à base lexicale française ? Etudes Créoles, 10.2, 60-75.

Baker, P., (ed.) (1995). From Contact to Creole and Beyond. Westminster : University of Westminster Press.

Beniak, E., \& Mougeon, R., (dir.), (1994). Les origines du français québécois. Sainte-Foy : PUL.

Bickerton, D., (1973). The nature of a Creole Continuum. Language, vol. 49, 640-669.

Bickerton, D., (1974). Creolization, linguistic universals, natural semantax and the brain. Working papers in linguistics, 6 , 126-41.

Bollee, A., (1978). Problèmes et perspectives de la description des créoles. Langue française, $\mathrm{n}^{\circ}$ 37, 21-39.

Brunot, F., (1935). Histoire de la langue française des origines à 1900. T. VIII, fasc. 2, 1117-1147.

Chaudenson, R., (1979). Les créoles français. Paris : Nathan.

Chaudenson, R., (2003). La créolisation : théorie, applications, implications. Paris : L'harmattan.

Chaudenson, R., (1994). Les créoles français sont-ils des formes simplifiées du français ? Cahiers du français contemporain, décembre ${ }^{\circ} 1,41-54$. F. Lapeyre, (éd.).

Chaudenson, R., (1995). Les français d'Amériques ou le français des Amériques ? Genèse et comparaison, Fournier R., et Wittmann H, 3-19.

Chaudenson, R., (1995). De la Vendée aux Caraïbes. Le Journal d'André Massé. Etudes Créoles, n 2, 97-110.

Cohen, M. \& Meillet, A., (1952). Les Langues du monde. Paris : CNRS.

Degraff, M., (1999). Language creation and language change: Creolization, diachrony and development. Cambridge: MA, MIT Press.

Degraff, M., (2001). Morphology. Creole genesis: Linguistics and ideology. Ken Hale: A life in language, ed. by Michael Kenstowicz, Cambridge, MA: MIT Press, 53-121.

Degraff, M., (2001). On the origin of Creoles: A Cartesian critique of 'Neo'-Darwinian linguistics. Linguistic Typology 5.2, 213-310.

Degraff, M., (2003). Against creole exceptionalism. Discussion note. Language, $\mathrm{n}^{\circ}$ 79, 391-410. 
Delumeau, F., (2006). Une description linguistique du créole guadeloupéen dans la perspective de la génération automatique d'énoncé. Thèse de doctorat soutenue à Paris X Nanterre.

Fattier, D., (1998). Contribution à l'étude de la genèse d'un créole: l'Atlas Linguistique d'Haïti, cartes et commentaires. Thèse de doctorat d'état, Université de Provence, T. 1-5.

Fon Sing, G., (2006). Du latin aux langues romanes, du français aux langues créoles: étude comparative de l'évolution et de la spéciation linguistique. Actes du colloque, Créolisation linguistique et sciences humaines. Paris : Presses Universitaires Haïtiano-Antillaises.

Galdi, L., (1949). De l'importance des parlers français-créoles pour la linguistique générale. Actes du $\sigma^{\text {ème }}$ congrès International de linguistes, Publié par Michel Lejeune. Paris : Klincksieck.

Goyette, S., (2000). From Latin to early Romance, a case of partial Creolization ? Language change and language contact in Pidgins and Creoles, 103-131 (éd) Mc Whorter.

Goyette, S., (2001). The emergence of the romance languages from latin. A case of creolization effects. Thèse de doctorat, Université d'Ottawa.

Hagège, C., (1985). L'homme de paroles. Paris : Fayard.

Hazaël-Massieux, G., (1996). Les créoles : problèmes de genèse et de description. Aix-en-Provence : Presses de l'Université de Provence.

Hazaël-Massieux, M.-C., (2005). Théories de la genèse ou histoire des créoles : l'exemple du développement des créoles de la caraïbe. La linguistique: les créoles. Revue de la Société Internationale de Linguistique Fonctionnelle, Vol.41-1, PUF.

Hazaël-Massieux, M.-C., (2008). Textes anciens en créole français de la Caraïbe: histoire et analyse. Paris : Publibook.

Jourdain, E., (1956). Du français aux parlers créoles. Paris : Klincksieck.

Lefebvre, C., (1998). Creole genesis and the acquisition of grammar. The case of Haitian Creole. New York : Cambridge university press.

Manessy, G., (1995). Créoles, pidgins, variétés véhiculaires. Procès et genèse. Paris : CNRS Editions.

Mintz S., (1971), "The socio-historical background to pidginization and creolization », Pidginization and Creolization of Languages, pp. 481-498.

Mufwene, S., (1986). Les langues créoles peuvent-elles être définies sans allusion à leur histoire ? Etudes créoles, vol. $I X, \mathrm{n}^{\circ} 1,135-150$.

Mufwene, S., (2005). Créoles, écologie sociale, évolution linguistique. Paris : L'harmattan.

Mufwene, S., (2002). Typologie des définitions des créoles. Linguistique et créolistique. Univers créoles 2. Paris : Economica.

Mufwene, S., (2005). L'étude des parlers créoles et son apport à la linguistique générale. Recherches Haïtianoantillaises, $\mathrm{n}^{\circ} 2$.

Mufwene, S., (2001). The Ecology of language evolution. New York : Cambridge University Press.

Mufwene, S., (2007). Les créoles: de nouvelles variétés indo-européennes désavouées? Actes du colloque: Créolisation linguistique et sciences humaines. Paris : Presses Universitaires Haïtiano-Antillaises.

Picoche, J., \& Marchello-Nizia, C., (1989). Histoire de la langue française, Paris : Nathan, (3 ${ }^{\text {ème }}$ éd. 1994).

Pinker, S., (1995). The Language Instinct. How the Mind creates Language. Harper Perrenial, (Prem. éd. 1994).

Pressoir, C. F., (1947). Débats sur le créole et le folklore. Port-au-Prince : Imprimerie de l'Etat.

Schleicher, A., (1852). Les langues de l'Europe moderne. Trad. Hermann E. Paris : Ladrange.

Schlieben-Lange, B., (1977). L'origine des langues romanes, un cas de créolisation ? Langues en contact-pidginscréoles, Tübingen : G. Narr. Meisel J. M., (éd).

Soutet, O., (2005). Linguistique. Paris : PUF. 
Valdman, A., (1976). Certains aspects sociolinguistiques des parlers créoles aux Antilles. Ethnies, n 3, 7-22.

Valdman, A., (1978). Le créole : structure statut et origine. Paris : Klincksieck.

Véronique, D., (2007). Ce que les langues créoles nous enseignent à propos de la genèse des langues. Actes du colloque : Créolisation linguistique et sciences humaines. Paris : Presses Universitaires Haïtiano-Antillaises.

Wartburg (von), W., (1946). Evolution et structure de la langue française. Berne : Franke.

Wartburg (von), W., (1946). Problèmes et méthodes de la linguistique. Paris : PUF.

Wittman, H., (1994). Grammaire comparée des variétés coloniales du français populaire de Paris du $17^{\text {ème }}$ siècle et origine du français québécois. Le français des Amériques, 281-334.

\footnotetext{
${ }^{1}$ Le lecteur nous excusera d'avance pour la longueur de certaine citation, mais nous avons préféré opérer en ce sens par souci de rendre la pensée de ses auteurs au plus près et afin qu'il ne puisse nous être reproché de déplacer les propos des auteurs de leur contexte.

${ }^{2}$ Il faut comprendre : éléments insérés dans la langue.

${ }^{3}$ Il faut comprendre : éléments disparus de la langue.

${ }^{4}$ Ibid., « le déterminisme du milieu intervient à deux moments dans l'évolution phonétique : au niveau de la langue originelle qui se dialectalise et au niveau des influences articulatoires liées à la langue ou aux langues antérieurement parlées sur ces lieux (dans le cas de l'émergence des créoles, il faudrait y inclure les langues sources de la population servile) », p. 50.

${ }^{5}$ Ibid., « le déterminisme structural comprend la stricte articulation phonétique et l'organisation de ces articulations en système (donc passage au point de vue phonologique) », p. 50.

6 Il y définit succinctement les koinés: 'these are compromise varieties from among diverse dialects of the same language'. P. 3
} 